\title{
Primary yolk sac tumour of the liver in adulthood
}

\author{
N A C S Wong, H D'Costa, R E Barry, D Alderson, M Moorghen
}

University of
Edinburgh, Teviot
Place, Edinburgh, UK:
Department of
Pathology
N A C S Wong
University of Bristol
and Bristol Royal
Infirmary, Bristol, UK:
Department of
Radiology
H D'Costa

Department of

Medicine

R E Barry

Department of
Surgery

D Alderson

\section{Department of Pathology and Microbiology \\ M Moorghen}

Correspondence to: Dr N A C S Wong,

Department of Pathology, University of Edinburgh, Edinburgh EH8 9AG, UK; email: nacs.wong@ed.ac.uk.

Accepted for publication 23 June 1998

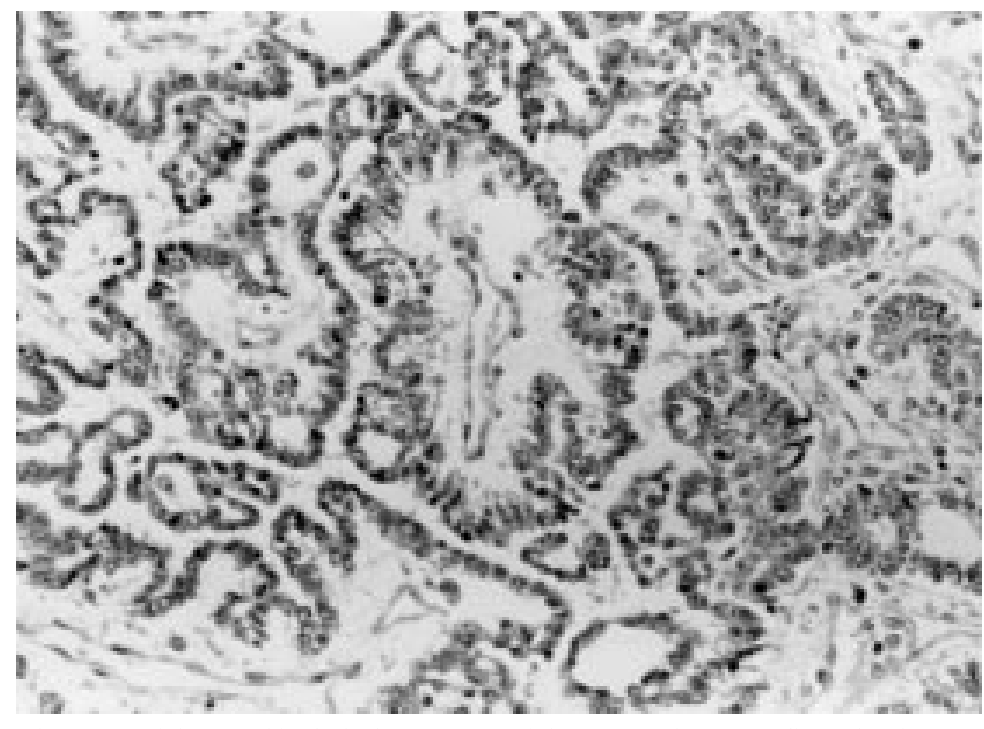

Figure 1 High power histological appearance of the tumour (haematoxylin and eosin, $\times 400$ objective). A Schiller-Duval body is arrowed.

Keywords: endodermal sinus tumour; liver neoplasms; $\alpha$-fetoprotein

Yolk sac (or endodermal sinus) tumours usually arise from the ovary or testis, though there have been reports of such tumours arising in extragonadal sites such as the mediastinum, bladder, and pineal gland. ${ }^{1}$ Primary yolk sac tumour of the liver was first reported by Hart in $1975 .^{2}$ Since then a further 12 cases have been reported, of which six presented in adulthood. ${ }^{13-7}$ Study of the present case and review of these six previous cases has revealed several characteristic features of adult primary yolk sac tumours of the liver which have not previously been commented on. These features may aid in the clinical distinction of the tumour

\begin{abstract}
Primary yolk sac tumour of the liver is exceedingly rare. A 28 year old woman presented with a cystic liver mass and a markedly raised serum $\alpha$-fetoprotein concentration. She underwent a partial hepatectomy for a suspected hepatocellular carcinoma but histological examination of the tumour revealed the classical morphological and immunohistochemical features of a yolk sac tumour. There was no evidence of an extrahepatic primary source. Review of this case, together with the six previously reported adult cases of primary yolk sac tumours of the liver, revealed several features of the tumour that may aid differentiation from hepatocellular carcinoma, with potential therapeutic implications.

(f Clin Pathol 1998;51:939-940)
\end{abstract}

nom and placental alkaline phosphatase. Neither immunoreactivity for hCG, a germ cell component, nor features of HCC or hepatoblastoma were seen. Despite the presence of infiltration into the attached diaphragm, excision of the tumour was complete. The background liver was normal. Postoperative clinical and radiological assessment, including transvaginal ultrasonography, showed no evidence of an ovarian or other extrahepatic tumour. At the time of writing (one year postoperatively), the patient remains well, with a normal serum $\alpha$-fetoprotein concentration and no sign of recurrence.

\section{Discussion}

The presentation of a young adult with a malignant, cystic tumour of the liver is unusual. The raised serum $\alpha$-fetoprotein concentration, in the absence of parenchymal hepatic disease, suggested a preoperative differential diagnosis 
Table 1 Reports of adult cases of primary yolk sac tumour of the liver

\begin{tabular}{|c|c|c|c|c|c|c|c|}
\hline Author (year) & Sex & Age (years) & Ethnic group & $a-F P(k U / l)$ & Size $(\mathrm{cm})$ & Location in liver & $\begin{array}{l}\text { Central } \\
\text { necrosis/ cystic } \\
\text { change } \|\end{array}$ \\
\hline $\operatorname{Yan}(1982)^{3}$ & $M$ & 58 & Oriental & 330000 & Multiple nodules $\dagger$ & Disseminated & $\ddagger$ \\
\hline Narita $(1982)^{5}$ & $\mathrm{~F}$ & 27 & Oriental & 23489 & $11 \times 11 \times 9.5$ & Right lobe & Yes \\
\hline Natori $(1983)^{4}$ & $\mathrm{~F}$ & 29 & Oriental & 3523 & $\begin{array}{l}\text { (1) } 17 \times 15 \times 10 \\
\text { (2) } 20 \times 25\end{array}$ & Right lobe & Yes \\
\hline Villaschi $(1991)^{6}$ & $\mathrm{~F}$ & 28 & European & 413 & $15 \mathrm{D}$ & Left lobe & Yes \\
\hline Whelan $(1991)^{1}$ & $\mathrm{~F}$ & 27 & Afro-Caribbean & 89000 & $15 \mathrm{D}$ & Right lobe & Yes \\
\hline Higuchi $(1993)^{7}$ & $\mathrm{~F}$ & 24 & Oriental & 115500 & "Large tumour" & Right lobe & Yes \\
\hline Wong $(1998)^{\star}$ & $\mathrm{F}$ & 28 & European & 14614 & $15 \times 15 \times 6$ & Right lobe & Yes \\
\hline
\end{tabular}

*This study.

tSize not specified.

$\ddagger$ Not commented on.

TCentral necrotic/cystic change detected by preoperative computed tomography.

$\alpha$-FP, serum $\alpha$-fetoprotein concentration on presentation.

of hepatocellular carcinoma, hepatoblastoma, or metastatic yolk sac malignancy. The latter was unlikely in view of the solitary nature of the liver tumour and the absence of any extrahepatic tumour. Regarding hepatoblastoma, this primitive tumour only rarely occurs in adults and usually presents as a solid mass. ${ }^{8}$

While this is only the seventh reported adult case of pure primary yolk sac tumour of the liver, there are several emerging correlates that may aid clinical distinction from hepatocellular carcinoma (table 1). Of the seven cases so far reported, six involved women aged between 24 and 30 years, ${ }^{13-7}$ whereas hepatocellular carcinomas show a male preponderance and usually present after the age of 50 years in Western countries. ${ }^{8}{ }^{9}$ It is uncommon for a hepatocellular carcinoma to present as a predominantly cystic tumour within a non-cirrhotic liver, as was seen in six of the seven cases. Finally, six of the seven cases showed a serum $\alpha$-fetoprotein concentration of more than $3000 \mathrm{kU} /$ litre, compared with only a minority of hepatocellular carcinomas. ${ }^{9}$

Distinguishing preoperatively between primary yolk sac tumours of the liver and hepatocellular carcinoma has important therapeutic implications. Hepatocellular carcinomas respond poorly to chemotherapy. ${ }^{9}$ While earlier reports of primary yolk sac tumours of the liver suggested a uniformly poor prognosis, ${ }^{5}$ Whelan and colleagues have more recently reported complete cure through chemotherapy followed by surgical resection. ${ }^{1}$ The diagnosis of primary yolk sac tumours of the liver was made preoperatively by computed tomography guided percutaneous liver biopsy, and following treatment with a combination of cisplatin, etoposide, and bleomycin, the resected lobe of liver showed no evidence of viable tumour and the patient remained disease-free five years after the operation. ${ }^{1}$ Our patient remains well with no evidence of tumour recurrence one year after surgery. However, as the long term outcome of surgically treated primary yolk sac tumours of the liver has yet to be established, our patient will continue to receive close clinical follow up.

In conclusion, primary yolk sac tumour of the liver, albeit rare, should always be considered as an alternative diagnosis to hepatocellular carcinoma in a young patient with grossly elevated serum $\alpha$-fetoprotein concentrations and a cystic tumour within a non-cirrhotic liver.

We thank Drs R L Jones and C P Case and Mr C P Barham for their contributions towards the assessment of this case.

1 Whelan JS, Stebbings W, Owen RA, et al. Successful treatment of a primary endodermal sinus tumour of the treatment of a primary endo
liver. Cancer 1992;70:2260-2.

2 Hart WR. Primary endodermal sinus (yolk sac) tumour of the liver. First reported case. Cancer 1975;35:1453-8.

3 Yan QH. Primary endodermal sinus tumour of the liver-a case report with a discussion on embryogenesis. Chung Hua Chung Liu Tsa Chih 1982;4:224-5.

4 Natori T, Teshima A, Kikuchi Y. Primary yolk sac tumor of the liver. An autopsy case with ultrastructural and immunopathological studies. Acta Pathol fpn 1983;33:555-64.

5 Narita T, Moriyama Y, Ito Y. Endodermal sinus (yolk sac) Narita T, Moriyama Y, Ito Y. Endodermal sinus (yolk sac)
tumour of the liver. A case report and review of the literatumour of the liver. A case repor

6 Villaschi S, Balistreri P. Endodermal sinus tumour of the liver. Histopathology 1991;18:86-8.

7 Higuchi T, Kikuchi M. Yolk sac tumour of the liver treated with transcatheter arterial embolization. Am f Gastroenterol 1993;88:1125-6.

8 Barwick KW, Rosai J. Liver. In: Rosai J, ed. Ackerman's surgical pathology, 8th ed. St Louis: CV Mosby, 1996:857942 .

9 Okuda K, Okuda H. Primary liver cell carcinoma. In: McIntyre N, Benhamou J-P, Bircher J, Rizzetto M, Rodes J, eds. Oxford textbook of clinical hepatology. Oxford: Oxford University Press, 1991:1019-53. 